\title{
A Randomized Pilot Clinical Assessment Of Three Skincare Regimens On Skin Conditions In Infants
}

This article was published in the following Dove Press journal:

Clinical, Cosmetic and Investigational Dermatology

\author{
Yuanyuan Duan (1D) \\ Lin $\mathrm{Ma}^{2}$ \\ Carlos Galzote $\mathbb{1}^{3}$ \\ Fan-Qi Kong' \\ Chun-Ping Shen ${ }^{2}$
}

'Johnson \& Johnson Asia Pacific Skin Testing Center, Minhang, Shanghai, People's Republic of China; ${ }^{2}$ Department of Dermatology, Beijing Children's Hospital, Capital Medical University, MOE Key Laboratory of Major Diseases in Children, Beijing, People's Republic of China; ${ }^{3}$ Johnson \& Johnson International (Singapore) Pte. Ltd., Singapore, Singapore
Correspondence: Carlos Galzote Johnson \& Johnson International (Singapore) Pte. Ltd., Km 14 Edison Road, Bo. Ibayo, Paranaque City 1700 ,

Philippines

Tel +6323688260

Fax +6328242329

Email cgalzote@its.jnj.com

\begin{abstract}
Introduction: Few data are available on the comparison between the effects on infant skin of skin care products and those of water alone.

Patients and methods: In this single-center, evaluator-blind, parallel-group pilot study, healthy infants were randomized to near-daily washing for 12 weeks (starting in the summer and finishing in the winter months) with a mild liquid baby wash followed by use of baby lotion (wash+lotion), water followed by baby lotion (water+lotion), or water alone. Clinical and instrumental assessments of skin moisturization and barrier function were made.

Results: As expected the skin condition in all groups was affected by the change of the season. The skin of infants in all groups was mildly deteriorated (clinical grading) and with reduced moisture levels and increased barrier function. Instrumental measurements indicated that skin moisture and barrier function were better maintained in the wash+lotion and water +lotion groups than in the water-only group at week 12. Clinical assessment scores increased slightly over 12 weeks in all groups $(P<0.05)$. At week 12 , the wash+lotion group $(\mathrm{n}=44)$ had significantly less change from baseline in overall skin condition and softness (lower scores $)$ than did the water+lotion $(n=43)$ or water-only $(n=43)$ groups. The wash+lotion regimen maintained stable erythema and rash scores with lower mean values over time than in the other groups.

Conclusion: A regimen of a liquid baby wash and a baby skin lotion for 12 weeks resulted in less detrimental changes in instrumental and clinical measures of skin than using water and lotion or water alone.
\end{abstract}

Keywords: skin abnormalities, erythema, exanthema, emollients, infant

\section{Introduction}

Infant's skin has a less developed epidermal barrier than adults, ${ }^{1-3}$ which emphasizes the importance of appropriate skincare routine to avoid the potential of skin problems. Water alone is insufficient to cleanse infant skin of water-insoluble impurities (e.g., components of saliva, nasal secretions, urine and feces), but some cleansers can impair skin barrier function by removing lipid components of the skin, thus increasing barrier permeability. ${ }^{4-6}$

Recent recommendations from a European roundtable meeting suggested that bathing infants is not harmful. ${ }^{7}$ Current recommendations suggest that liquid cleansers formulated for babies are noninferior to washing with water, ${ }^{8-10}$ and that washing with appropriately formulated cleansers does not impair the skin barrier, $^{7,8}$ and is preferable to using water alone. ${ }^{9,11}$ Unlike adult cleansers, good baby cleansers are mainly composed of non-ionic and amphoteric surfactants that aggregate to form larger micelles. The large micelle surfactant system makes baby 
cleansers milder as larger micelles are less likely to penetrate the skin and disrupt the protective skin barrier. ${ }^{4}$

Skin that is too dry can lead to skin barrier disruption and even infection. ${ }^{21}$ Compared with adults, infant skin is particularly vulnerable to dryness due to smaller corneocytes, larger gaps between corneocytes, thinner stratum corneum, fewer normal microflora and less skin lipid. ${ }^{19,22,23}$ Hence, infant skin is more vulnerable to more than short-term changes in the environment (temperature and humidity), such as season change. ${ }^{1}$ Moisturizing (emollient) lotion preserves, protects and enhances the baby's skin barrier by supplying the stratum corneum with water and lipids, and creates a protective barrier on baby skin to inhibit water loss; thus, emollients have been recently recommended for use to improve and maintain the skin barrier. ${ }^{7,12,13}$

Systematic review of studies with the use of cleanser and/or emollient in infants highlights the requirement for more studies. ${ }^{8,14,15}$ Infant skin continues to develop during the first year after birth,,3 which makes an unstable baseline for studies comparing wash regimens. ${ }^{8,16}$ Few randomized controlled studies have investigated the use of a cleanser and/or moisturizer regimen on healthy, full-term infants over a period of months. ${ }^{9,17,18}$ The objective of this pilot study was to investigate the effects of three different skincare regimens, applied over 12 weeks, in infants (aged 0-6 months at the start of the study). Outcome measures included clinical dermatological assessments, instrumental assessments of skin barrier properties, and parent/caregiver assessments.

\section{Materials And Methods}

\section{Trial Design}

This was a single-center (Beijing Children's Hospital, Xicheng District, China), clinical evaluator-blind, parallel pilot study in healthy, full-term infants (aged 0-6 months at study entry). Recruitment age was modified after the start of the study from the original protocol definition of 5-6 weeks due to low initial recruitment.

\section{Participants}

Potential participants were identified by the use of an advertisement placed within local maternity hospitals. The advertisement targeted pregnant women who had regular antenatal examinations within these hospitals. Parent/ legal guardian was pre-screened by telephone using an approved script and had to be willing to commit to prespecified trial conditions, including a daily log of events. Infants with pre-existing medical conditions or familial history of conditions that may adversely affect the outcome of the study were excluded. Only one infant per household could enter the study. Inclusion and exclusion criteria for study participants (both infant and parent/legal guardian) are shown in Table 1.

Clinical and instrument assessments were carried out at the study center during scheduled visits, during which parent/legal guardian assessments were also reviewed by investigators. Institutional review board approval (Beijing Children's Hospital, Capital Medical University) and written informed consent from each child's participating parent and/or legal guardian were obtained before enrollment of an infant into the study. (www.ClinicalTrials.gov, NCT02981056). The study was conducted in accordance with the ethical principles of the Declaration of Helsinki.

\section{Skincare Treatment Regimens}

After screening by the investigators at the Beijing Children's Hospital, infants meeting inclusion criteria were allocated into three treatment groups. The parent/legal guardian

Table I Inclusion And Exclusion Criteria

\begin{tabular}{|l|l|}
\hline Inclusion Criteria & Exclusion Criteria \\
\hline - Healthy full-term infant (0-6 months old) & $\begin{array}{l}\text { Pre-existing skin conditions (dermatitis, eczema, psoriasis, rosacea), } \\
\text { dry skin } \\
\text { - Male or female }\end{array}$ \\
$\begin{array}{l}\text { - Parent/caregiver } \geq 18 \text { years old } \\
\text { - Willingness of parent/caregiver to follow study instructions and sign } \\
\text { - Willingness of parent/caregiver to avoid prolonged exposure of infant } \\
\text { to sun, beach, or swimming pool }\end{array}$ & $\begin{array}{l}\text { impact results (except vitamins) } \\
\text { allergic response to skin care products }\end{array}$ \\
- Willingness of caregiver to attend all scheduled visits & $\begin{array}{l}\text { Participant with active localized or general infection } \\
\text { Other condition which may make the subject inappropriate for trial } \\
\text { entry }\end{array}$ \\
\hline
\end{tabular}

Note: Screening for inclusion included taking a medical history, obtaining informed consent and performing baseline assessments. 
received test material along with detailed verbal and written instructions for product use, and a diary in which to note study events as specified by verbal and written instructions provided.

Group 1: Mild liquid baby wash + baby lotion (Johnson's ${ }^{\circledR}$ Baby Top-To-Toe ${ }^{\circledR}$ Wash and Johnson's ${ }^{\circledR}$ Baby Lotion, Johnson \& Johnson Consumer Inc., Skillman, NJ; wash +lotion). Babies were cleansed five to seven times a week and lotion were applied at least once (not more than twice) per day. Directions included dispensing wash into the palm of the caregiver's hand and gently lathering. Using only hands, the caregiver gently cleansed the baby's hair and body, avoiding the eyes. The baby was gently rinsed with water and toweled dry. Lotion was applied to the baby's face, arms, legs, and torso (avoiding eyes and diaper area) and smoothed into the skin.

Group 2: Water (in lieu of baby wash) + baby lotion (Johnson's ${ }^{\circledR}$ Baby Lotion, Johnson \& Johnson Consumer Inc., Skillman, NJ; water+lotion). In this group, the baby was cleansed with water only. After the infant was gently rinsed with water and toweled dry, lotion was applied at least once a day.

Group 3: Water (in lieu of baby wash and lotion). In this group, the baby was cleansed with water only, as above, and lotion was not used.

\section{Assessments}

The primary outcomes of this study, which included skin subsurface hydration and water content, were measured using a Moisture Meter- $\mathrm{D}^{\circledR}$, Skin surface moisture content was monitored via capacitance (Corneometer ${ }^{\circledR}$ 825, Courage \& Khazaka, Köln, Germany) and the transepidermal water loss (TEWL) by Vapometer ${ }^{\circledR}$. Secondary endpoints included skin roughness, skin $\mathrm{pH}$, dermatological assessment, and parental assessment. As this was a pilot study, the trial was not powered to detect statistical differences between study groups.

Clinical and instrumental assessments were made in the clinic at three scheduled study visits: baseline, week 6, and week 12. Assessments were made after a 30-min acclimatization period in the climate-controlled clinic (set at $20-24^{\circ} \mathrm{C}$ and $40-60 \%$ relative humidity). If the infant was visibly active (eg, crying, distressed, playing) during the assessment, the assessor had to wait for re-acclimatization. The parent/legal guardian maintained a home diary and completed questionnaires about the use of the test products at each clinic visit.

\section{Instrumental Assessments}

Skin roughness was estimated from digital images (Skin Evidence ${ }^{\mathrm{TM}}$ Pro, INTUISKIN SA, Grenoble, France) with skin imaging at $50 \times$ magnification on both forearms near the wrist. $^{19}$

Skin surface moisture content was monitored via capacitance (Corneometer ${ }^{\circledR}$ 825, Courage \& Khazaka, Köln, Germany). Measurements were taken on both inner forearms close to the elbows. ${ }^{20}$

Skin subsurface hydration and water content were measured using a Moisture Meter-D ${ }^{\circledR}$ (Delfin Technologies Ltd., Kuopio, Finland), which measures to a depth of 500 microns, on both inner forearms close to the elbows. ${ }^{21,22}$

Skin $\mathrm{pH}$ was measured on the inner forearms close to the wrists (Skin pH Meter PH900, Courage \& Khazaka).

Transepidermal water loss measurements were taken using a Vapometer ${ }^{\circledR}$ (Delfin Technologies); lower readings indicated better skin barrier function. One measurement was taken on each of the inner forearms. ${ }^{23}$

\section{Clinical Assessments}

A clinician evaluated each infant's face, arms (both combined), legs (both combined), and torso (chest and back combined) for overall skin condition, softness, dryness, redness (erythema), rash (irritation), and tactile roughness. Every effort was made to have the same clinician perform all clinical assessments at every visit. Overall skin condition and softness were scored using a 5-point scale, with half-point scores used as necessary, with $0=$ excellent, $1=$ very good, $2=$ good, $3=$ fair, and $4=$ poor. Dryness, erythema, rash and tactile roughness were scored on a four-point scale, with half-point scores used as necessary: $0=$ none, $1=$ mild, $2=$ moderate, and $3=$ severe (Supplementary Table 1). Composite mean scores were calculated from the individual scores for face, arms, legs and torso.

\section{Parent/caregiver}

Parent/legal guardian completed a questionnaire at baseline, week 6 and week 12 rating the current skin condition on face, arms, legs and torso of their infant using the same 5 -point scale $(0=$ excellent; $4=$ poor $)$ as above. Composite mean scores were calculated.

\section{Sample Size, Randomization And Blinding}

Each of the three treatment groups was estimated to require 30 infants, which is a broadly accepted industry standard as the minimum number of subjects to 
demonstrate statistical significance. In anticipation of a dropout rate that may be higher than normal because of the long duration of the study $(\sim 3$ months $)$, more subjects were recruited.

Infants were randomized by computer to each group with stratification according to age and to achieve an approximately $1: 1$ ratio of males to females, vaginal to caesarean section delivery, and those living in urban versus nonurban locations, and infant ages were allocated evenly.

The instrument assessor, dermatologist/grader and reviewer of parent/legal guardian diary and questionnaire were blinded to the treatment regimens received by the infant. The parent/guardian was open to the treatment regimen received by the infant, but blind to the products used.

\section{Data Analysis}

As this was a pilot study, the analysis was designed to provide estimates of future trial parameters. As such, no formal sample size calculation or formal statistical comparisons between study groups were made. Standard normality (Kolmogorov-Smirnov) and homogeneity-of-variance (Levene) tests were performed to determine the parametric data type for each instrumental and subjective assessment.
To determine whether the differences between baseline and subsequent readings (week 6 and week 12) were significant, one-way analysis of variance (Dunnett's multiple comparison, for parametric data) and $\mathrm{K} / 2$ related samples test (Friedman/Wilcoxon, for nonparametric data) were used. A significance probability of $P<0.05$ was used.

\section{Results}

\section{Subjects}

A total of 150 infants were enrolled and 130 completed the study. Twenty infants discontinued by parental request (Figure 1). Subject demographics are shown in Table 2.

\section{Environmental Assessment}

Studies began in June 2013 and continued through November 2013 in Beijing, China. Temperature and humidity varied over this timeframe, with outdoor temperatures falling below inside temperatures beginning in September (Figure 2).

\section{Instrumental Assessments}

Using two different methods (Corneometer and the Moisture Meter-D), the moisture content in the skin was found to decrease over time in all groups by week 12 (Figure 3A and C). Corneometer measurements signaled

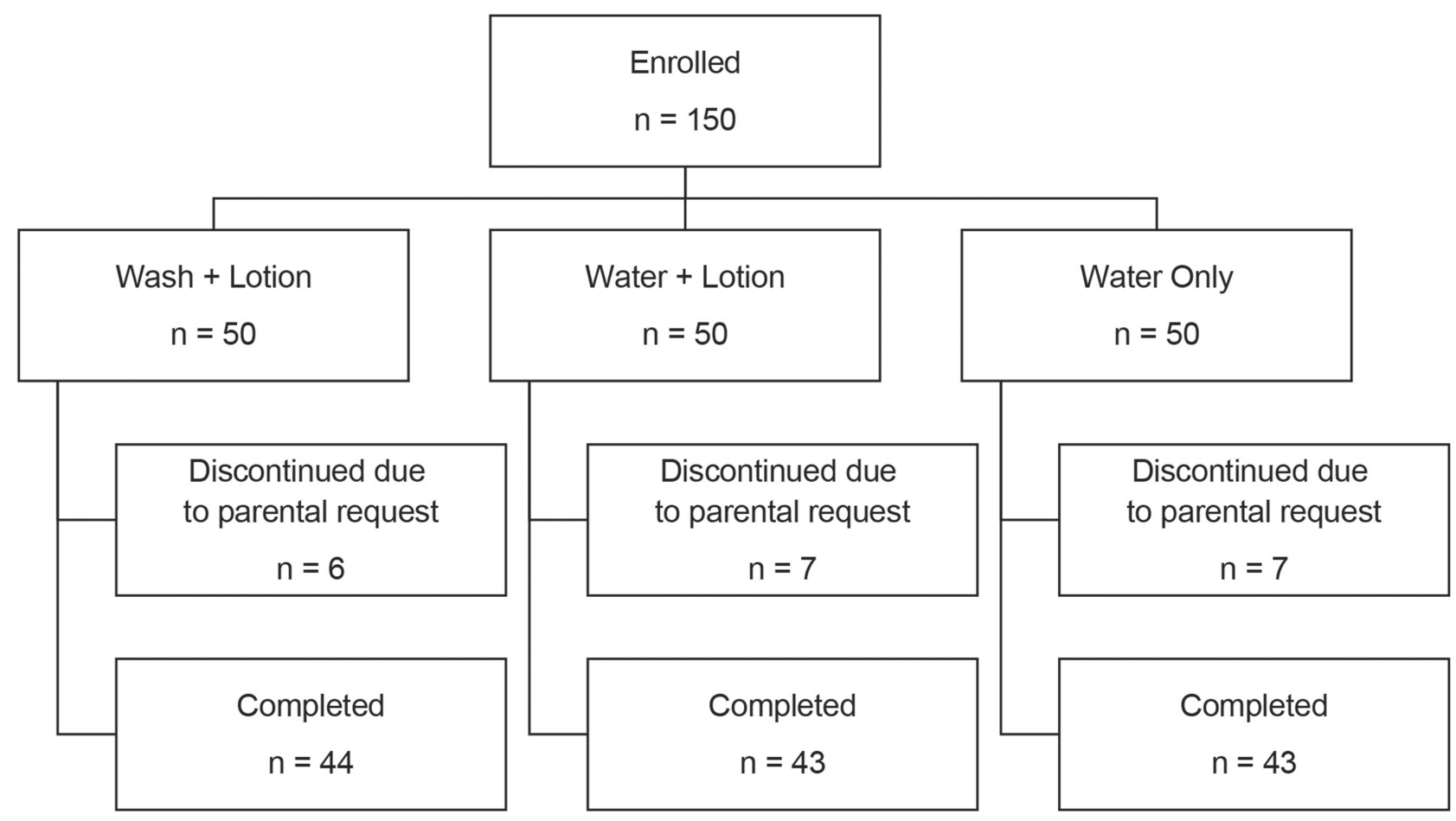

Figure I Patient flow through the trial. 
Table 2 Subject Demographics

\begin{tabular}{|c|c|c|c|c|}
\hline & Wash+lotion & Water+lotion & Water Only & Total \\
\hline Number of completed subjects & 44 & 43 & 43 & 130 \\
\hline Age in days, mean $\pm S D$ & $110.93 \pm 45.98$ & $105.44 \pm 49.33$ & $107.47 \pm 54.12$ & $107.97 \pm 49.56$ \\
\hline \multicolumn{5}{|l|}{ Sex } \\
\hline Male, n (\%) & $24(54.5 \%)$ & $29(67.4 \%)$ & 17 (39.5\%) & 70 (53.9\%) \\
\hline Female, $\mathrm{n}(\%)$ & $20(45.5 \%)$ & $14(32.6 \%)$ & $26(60.5 \%)$ & $60(46.1 \%)$ \\
\hline \multicolumn{5}{|l|}{ Delivery type } \\
\hline Vaginal delivery, $\mathrm{n}(\%)$ & $21(47.7 \%)$ & $20(46.5 \%)$ & $25(58.1 \%)$ & $66(50.8 \%)$ \\
\hline Caesarean section, $\mathrm{n}(\%)$ & $23(52.3 \%)$ & $23(53.5 \%)$ & 18 (4I.9\%) & $64(49.2 \%)$ \\
\hline \multicolumn{5}{|l|}{ Residential type } \\
\hline Urban, n (\%) & $36(39.6 \%)$ & 27 (29.7\%) & $28(30.8 \%)$ & 91 (70.0\%) \\
\hline Rural, n (\%) & $8(20.5 \%)$ & $16(41.0 \%)$ & $15(38.5 \%)$ & $39(30.0 \%)$ \\
\hline
\end{tabular}

a decrease in moisture content in the water-only regimen as early as 6 weeks (Figure 3A). The water-only regimen was associated with a greater change from baseline in skin moisture at week 12 than was observed in the other groups

A
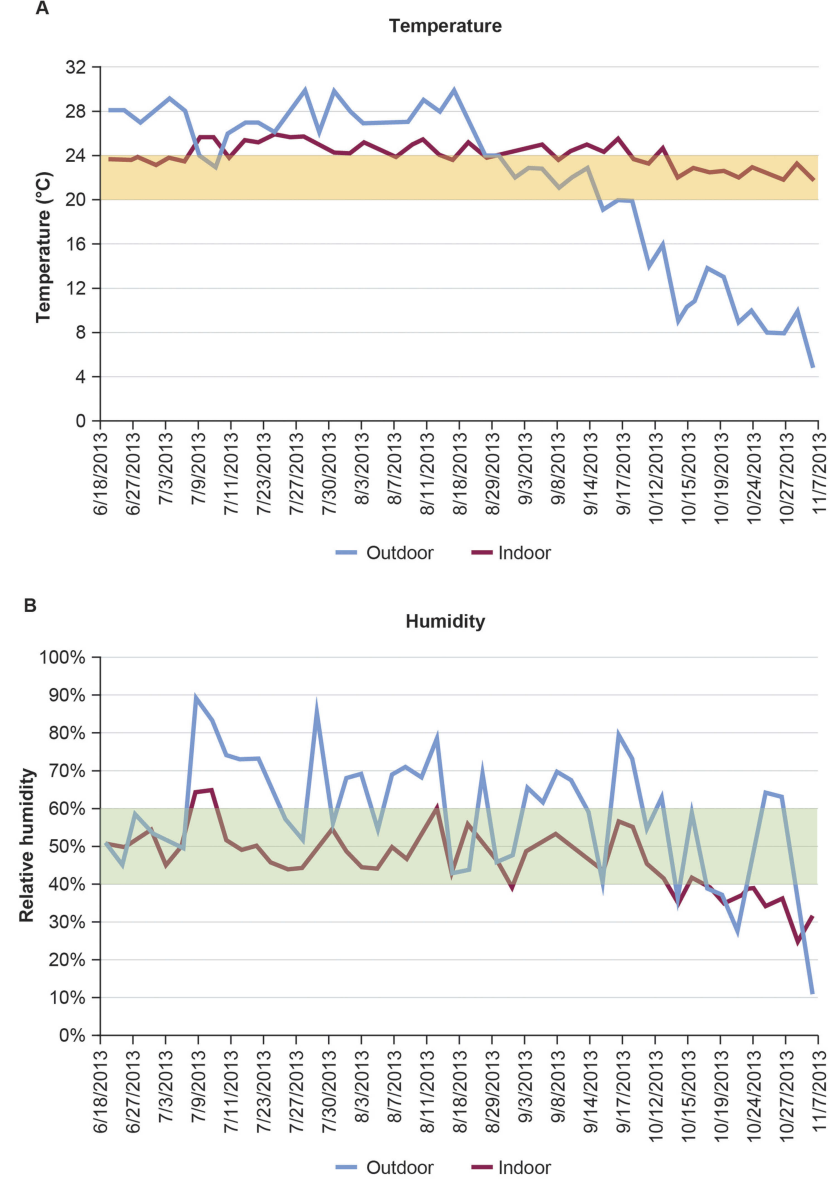

Figure 2 (A) Temperature and (B) humidity profile over the course of the study. Note: Blue lines indicate outside temperature and humidity and red lines indicate inside values.
( $P<0.05$; Figure 3B). Using the Moisture Meter-D to measure subsurface moisture demonstrated that at 12 weeks, differences in the change from baseline in moisture between the wash+lotion and water+lotion groups were not seen, but a statistically significant reduction occurred in subsurface moisture in the water-alone group compared with the water+lotion group (Figure 3D). Taken together, these findings suggest that incorporating a lotion application during the cleansing routine, done with water or with a mild cleanser, was associated with better maintenance of skin moisture than cleansing with water alone.

After 12 weeks of intervention, all groups showed a continuous and statistically significant decrease in TEWL (improved skin barrier function; Figure $4 \mathrm{~A}$ and $\mathrm{B}$ ). These results indicate that the wash+lotion regimen was more effective in preserving the skin barrier than either water+lotion or water alone.

Skin $\mathrm{pH}$ was lower (more acidic) in the wash+lotion group at week $6(P<0.05$; Figure 5A). The decrease from baseline in skin $\mathrm{pH}$ was also greater in the wash+lotion regimen than water+lotion and water alone at 6 weeks $(P<0.05$; Figure 5B). These results suggest that the wash +lotion regimen may be more effective in helping to maintain a low skin $\mathrm{pH}$; further validation is needed.

Skin roughness, measured optically, generally increased over the 12-week experimental period (Figure 5C). However, roughness in the wash+lotion group was less notably increased over time and appeared to have a smaller change from baseline than the other groups (Figure 5D).

\section{Dermatologist Assessments}

No change from baseline in overall skin condition was indicated between groups at week 6 (Figure 6A). At week 12 , the change from baseline in the water+lotion 


\section{Corneometer}

A

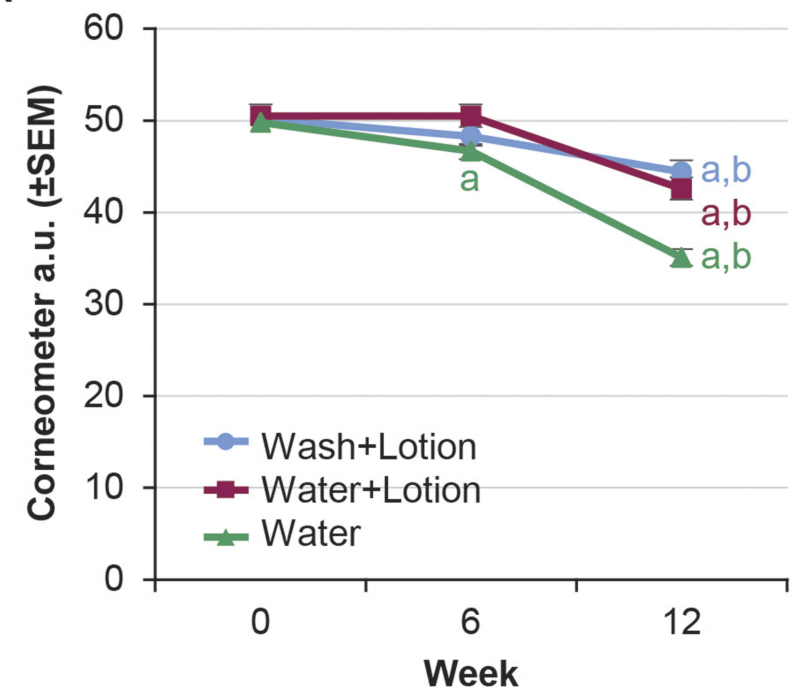

B

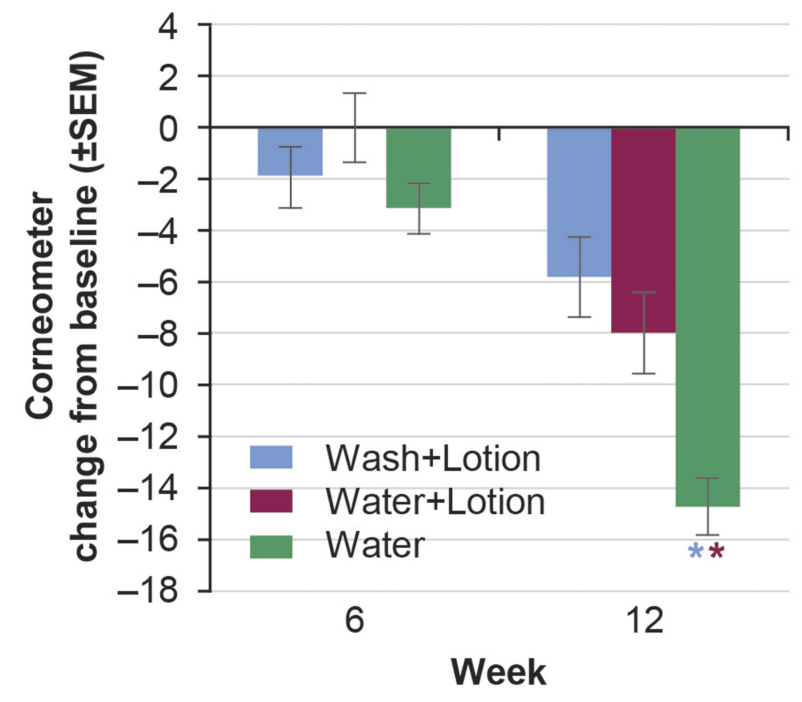

Moisture Meter-D

C

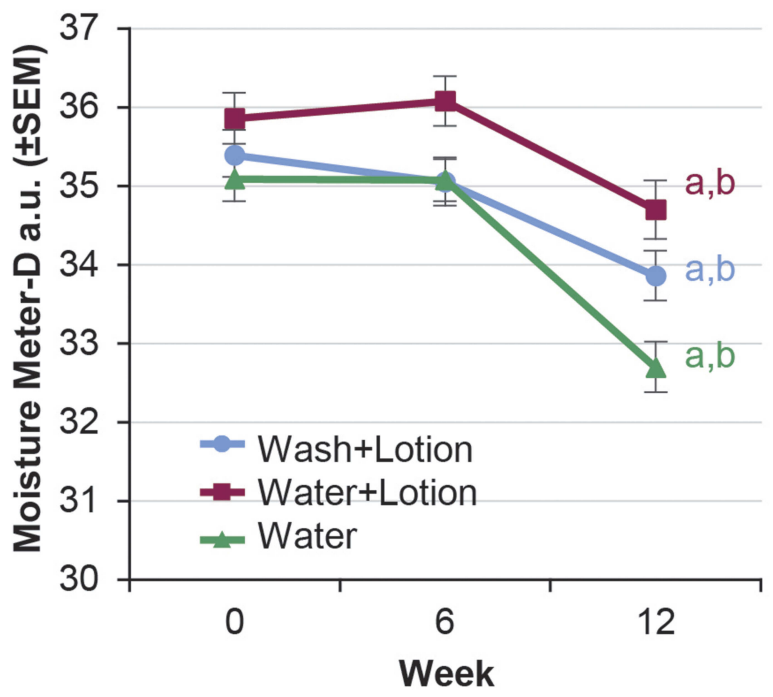

D

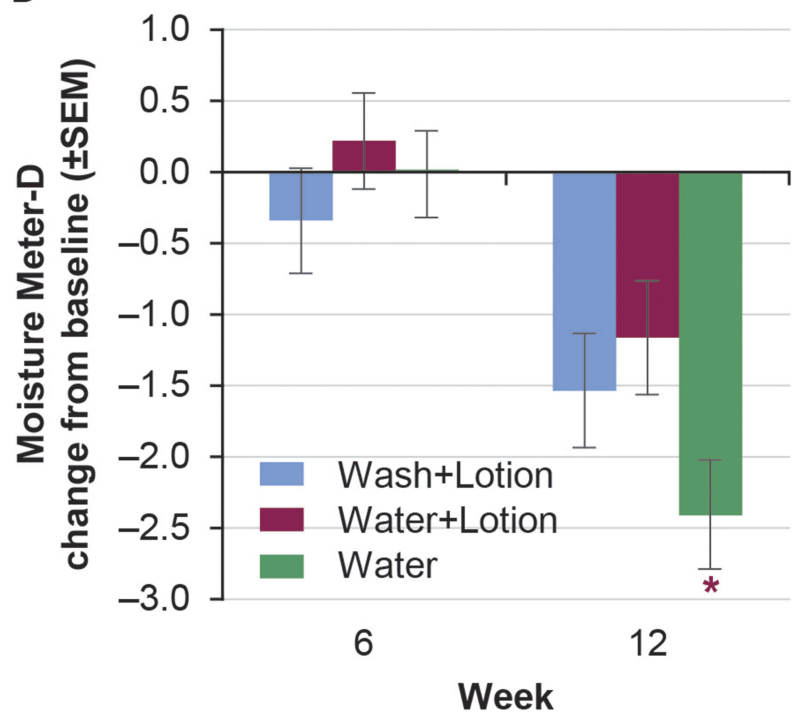

Figure 3 Skin moisture. (A) Corneometer value over time and (B) change from baseline in Corneometer value. (C) Moisture Meter-D value over time and (D) change from baseline.

Notes: ${ }^{\text {a }} P<0.05$ vs week $0,{ }^{b} P<0.05$ vs week $6 . * P<0.05$. Color of asterisk over bar signifies the specific comparison made.

Abbreviations: SEM, standard error of the mean; a.u., arbitrary units.

or water-only groups was greater than in the wash +lotion group $(P<0.05)$. No differences were observed between water+lotion and water-only at week 12 (Figure 6A).

In the dermatologist assessment of skin softness, the wash+lotion group had a lower (softer skin) score at week $6(P<0.05)$. At week 12, water+lotion and wateronly had greater change from baseline in skin softness scores than wash+lotion $(P<0.05$; softest in the wash +lotion group). No changes from baseline in softness at week 12 were observed between the water+lotion and water-only groups (Figure 7A).

Dryness increased in all groups over time. At week 6 , the water-only group had a greater change from baseline (more dryness) than the wash+lotion and water +lotion groups (Figure 8A). By week 12, there were no changes from baseline indicated in dryness between groups (Figure 8A). 


\section{TEWL}

A

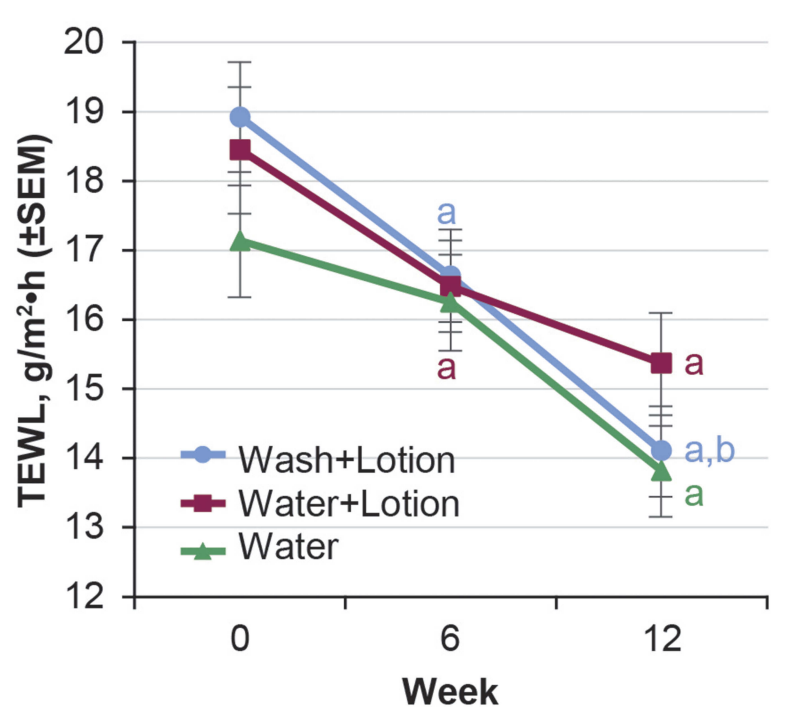

B

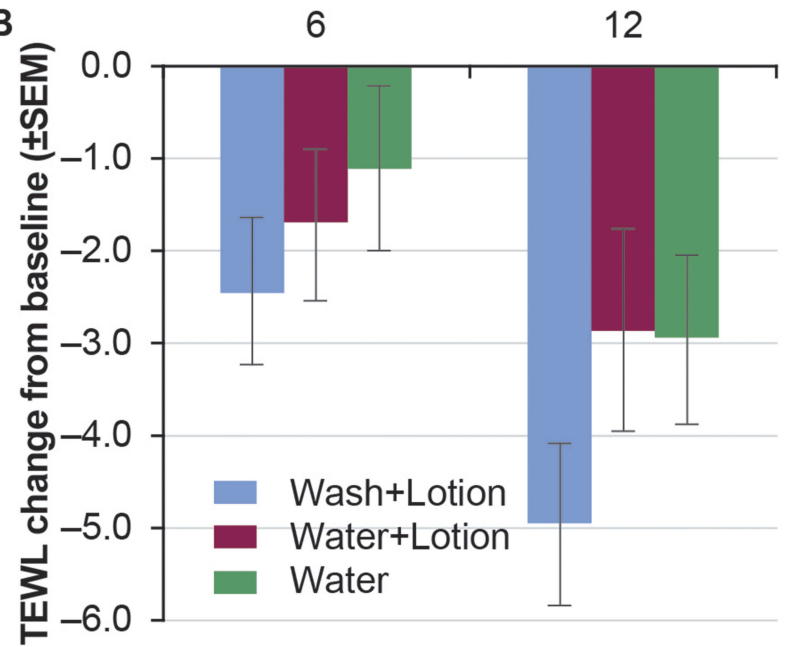

Week

Figure 4 Skin barrier function. (A) TEWL over time and (B) Change in TEWL from baseline.

Notes: ${ }^{a} P<0.05$ vs week $0,{ }^{b} P<0.05$ vs week 6 . Color of letters over lines signifies the specific comparison made.

Abbreviation: SEM, standard error of the mean.

Redness/erythema was not significantly different from baseline at week 12 in any group. Perhaps because of a greater baseline value in the water-only group, the decrease in erythema in this group was significantly greater than the other groups at week 6 , yet no changes from baseline between groups were indicated at week 12 (Figure 9A). Rash/irritation also was not noted to vary over time. No difference between the groups in change from baseline in rash/irritation was noted (Figure 10A). Mean values for rash/irritation and redness/erythema in the wash+lotion group appeared less variable across the time points than in the other two groups.

Tactile roughness increased in all groups at week 6 and week 12 . The water-only group had greater change from baseline than wash+lotion or water+lotion groups at week $6(P<0.05$; Figure 11A); however, no changes from baseline were indicated among groups at week 12 .

\section{Parent/caregiver Assessments}

Except for overall skin condition (Figure 6B) and softness (Figure 7B), parent/caregiver assessments showed more improvement over time than the study dermatologist evaluator assessments (Figure 6-11, part C) despite the shared rating scale. These results showed that more babies had skin evaluated with changes in dryness, redness, rash/irritation and roughness by dermatologists than that by parents, especially during colder and drier seasons. This shows that there is a difference between perception and reality, and baby skin is evaluated less well by parents than by a dermatologist, as might be expected since dermatologists are trained to evaluate skin at a discriminating level. Additionally, parents expecting positive changes in skin texture as study participants may perceive positive changes.

\section{Discussion}

The results from this study indicate that a regimen of bathing the infant with a mild liquid baby cleanser, followed by a moisturizer developed especially for baby skin, was gentler to the skin than tap water alone. Instrumental analyses confirmed the clinical findings, particularly in terms of maintaining skin moisture, improving skin barrier function, and possibly regulating skin $\mathrm{pH}$.

The increase in clinical skin dryness over time observed in this study may also reflect the seasonal decrease in outdoor temperature and humidity during the study period. The study started in June (hot and humid) and ended in mid-November (cold, dry and windy) in Beijing, China. Many of the clinical assessments were similar among treatments for the first half of the study, corresponding to the warmer months. Once the season changed and outdoor temperatures and humidity began to 
$\mathrm{pH}$

A

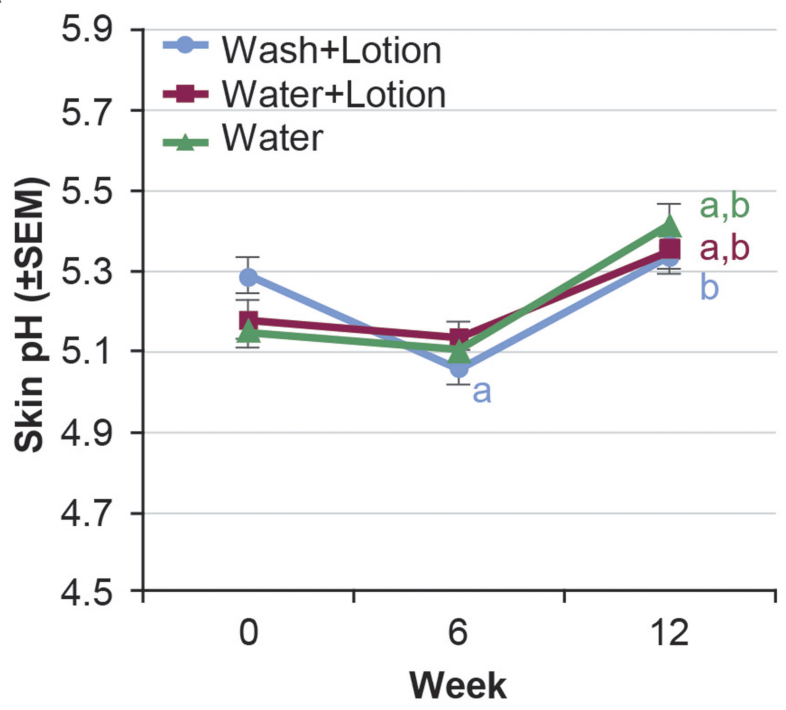

B

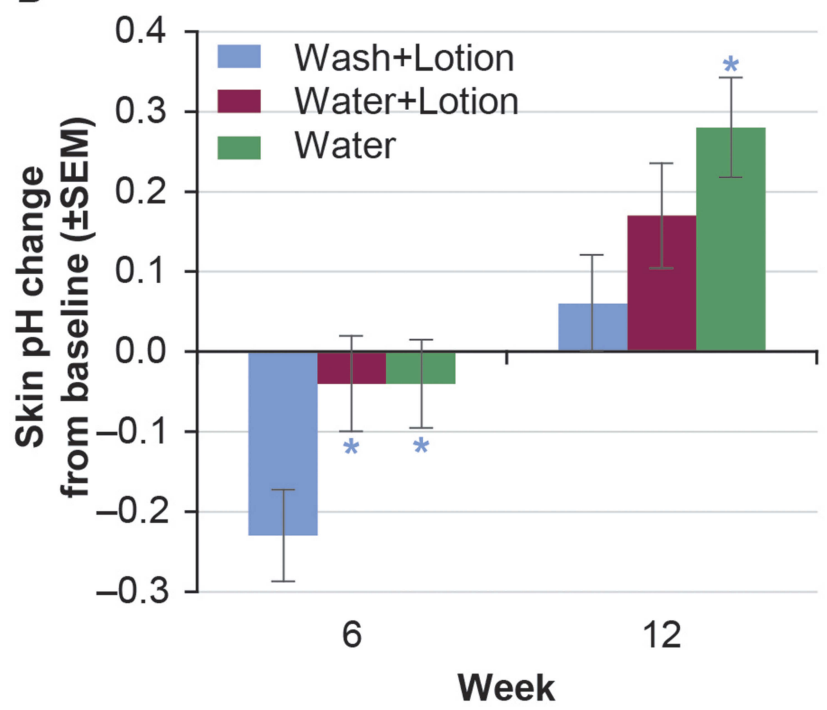

Roughness (image analysis)

C
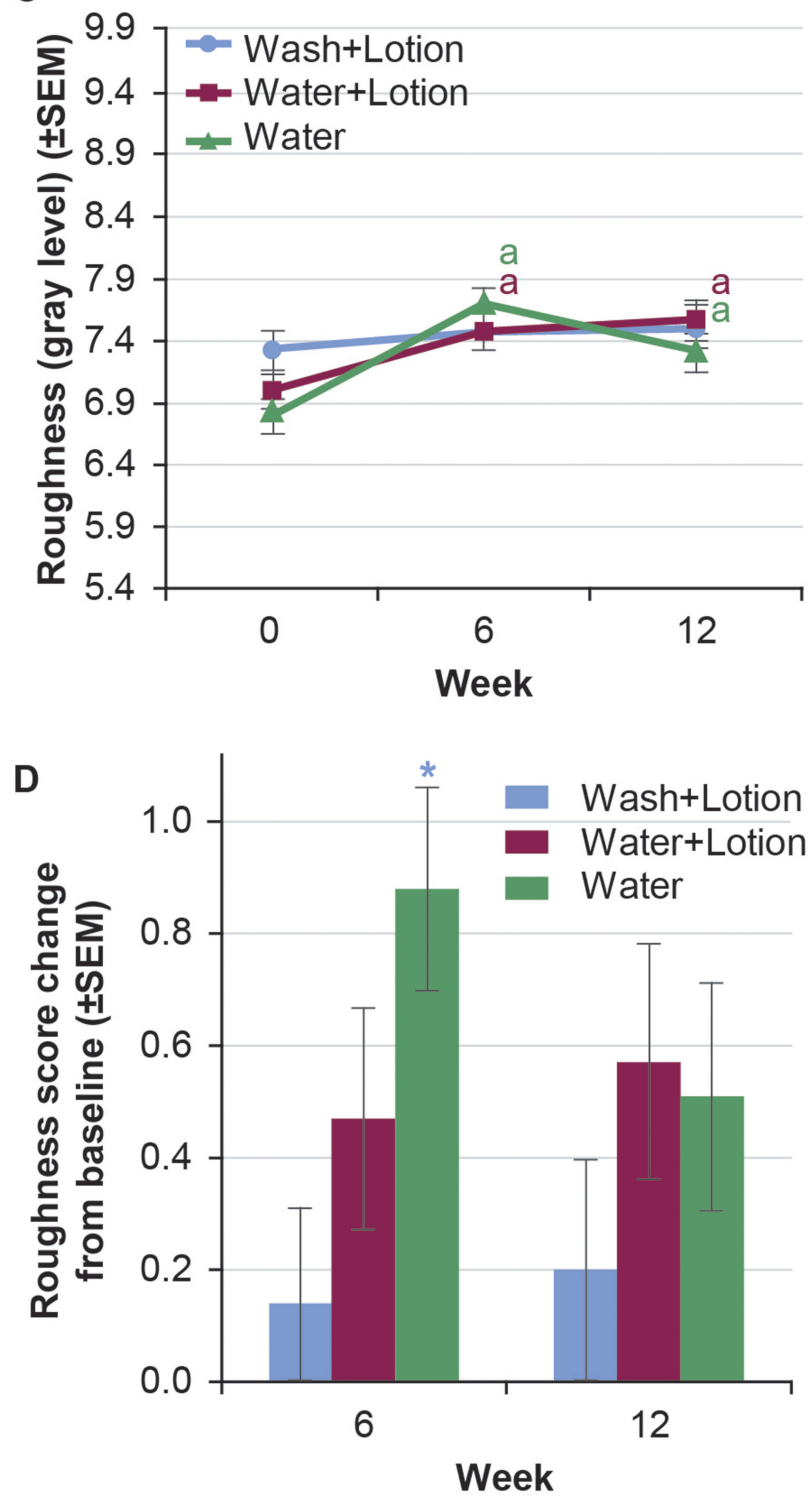

Figure 5 Skin $\mathrm{pH}$ and skin roughness. (A) Skin $\mathrm{pH}$ over time, (B) skin $\mathrm{pH}$ change from baseline, (C) skin roughness score (grey level, SkinEvidencePro) from images of forearm skin, and (D) skin roughness change from baseline.

Notes: ${ }^{a} P<0.05$ vs week $0,{ }^{b} P<0.05$ vs week $6 .{ }^{*} P<0.05$. Color of asterisk over bar signifies the specific comparison made.

Abbreviation: SEM, standard error of the mean.

decrease, clinical measures of softness, dryness, and irritation began to worsen in all groups.

Conducting our study in a more temperate climate might have produced different results. Local climate was concluded to affect conductance and TEWL in a comparative study between infants located in Beijing, Mumbai (India) and Skillman (New Jersey, USA). ${ }^{1}$ In the same study, children from Beijing were observed to have a higher incidence of facial scaling and erythema than similarly aged children living in Mumbai, ${ }^{1}$ and these differences were also attributed to the colder and dryer environment in Beijing in the winter, when the measurements were made.

Assessments by parents/guardians and dermatologists were in general agreement with each other, although none of the mean numerical scores showed major changes. One difference of note was that dermatologists evaluated more babies to have dry skin than evaluated by parents, which 


\section{Dermatologist}

A

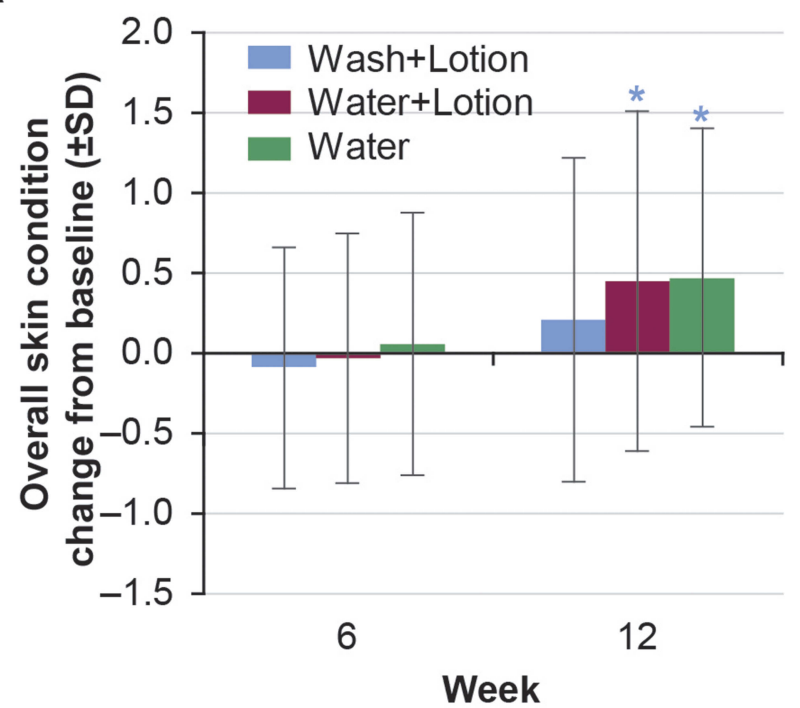

\section{Parent/Caregiver}

B

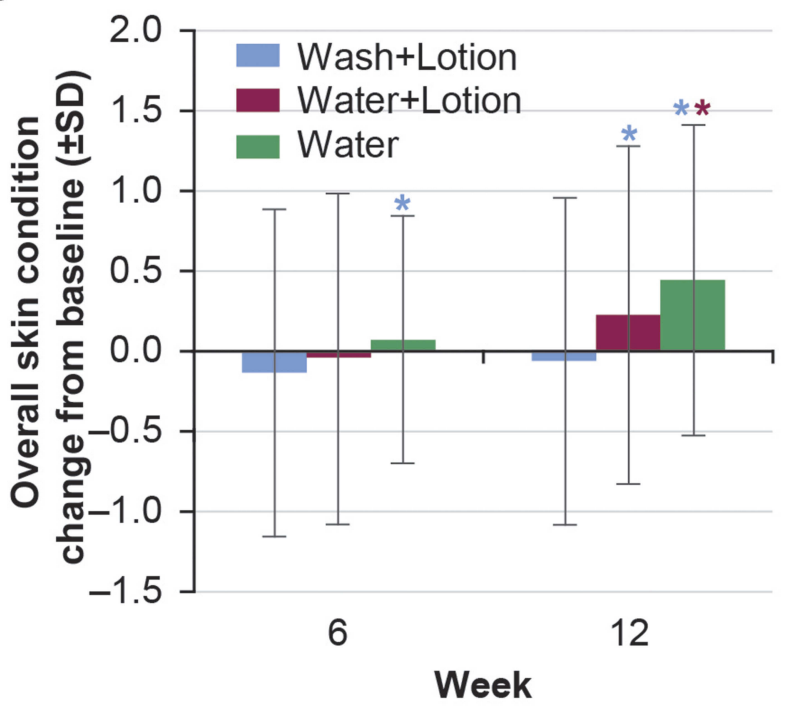

C

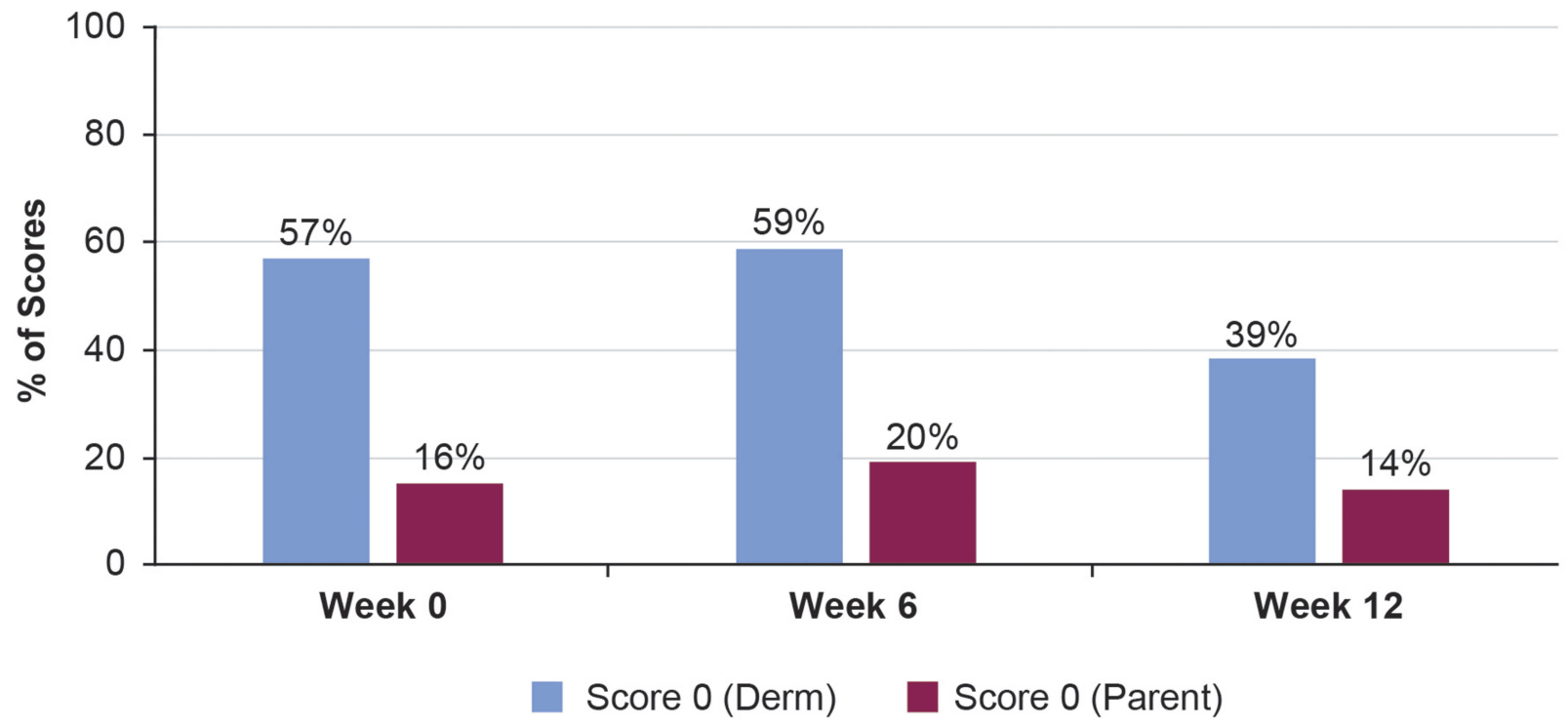

Figure 6 Overall skin condition. Dermatologist evaluation of overall skin condition score change from baseline (A). Parent/caregiver scores change from baseline (B). Comparison between dermatologist or parent scoring 0 at Week 0, 6 and 12 (C).

Notes: $* P<0.05$. Color of asterisk over bar signifies the specific comparison made.

Abbreviation: SD, standard deviation.

indicates that baby skin can be drier than it looks. This indicates the need for a higher level of hydration for baby skin, which can be supported with moisturization. Mean clinical scores did not exceed a score of 1.5 for any category, and skin condition would have been rated as "good" or "mild" after each regimen.

After birth, the skin continues to develop. ${ }^{1-3,24}$ TEWL is thought to be high after birth and then to decrease with age, becoming similar to that seen in the adult after 3 to 4 years, ${ }^{2}$ an effect noted in several different ethnic groups, including in a group of children from Beijing. ${ }^{1}$ Similar trends have also been noted with skin conductance. ${ }^{1,3}$ Taken together, these reports suggest that the skin barrier in infants is more permeable and less resilient than in adults. For these reasons, recent guidelines have suggested procedures to prevent skin barrier disruption in infants. ${ }^{7,25}$ 


\section{Dermatologist}

A

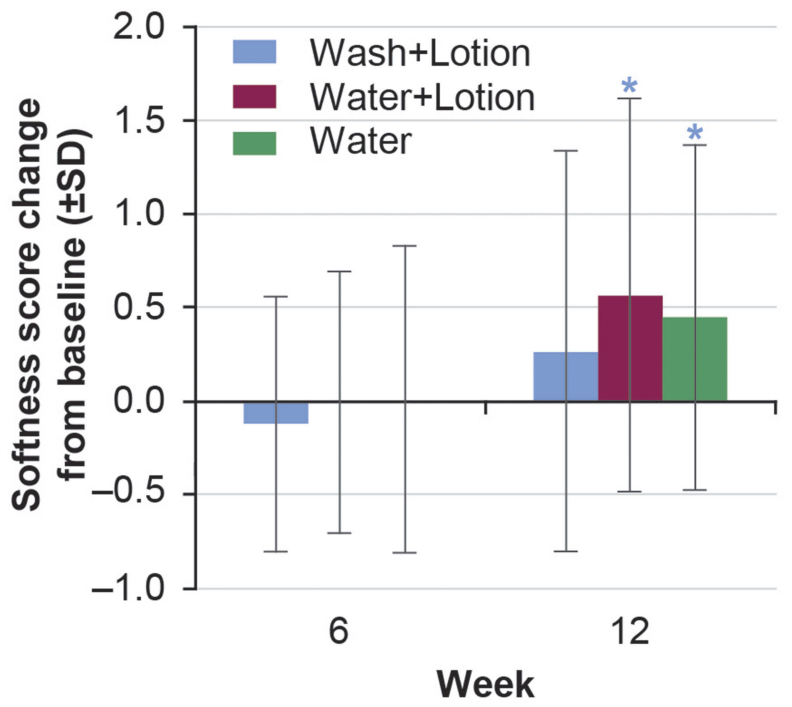

Parent/Caregiver

B

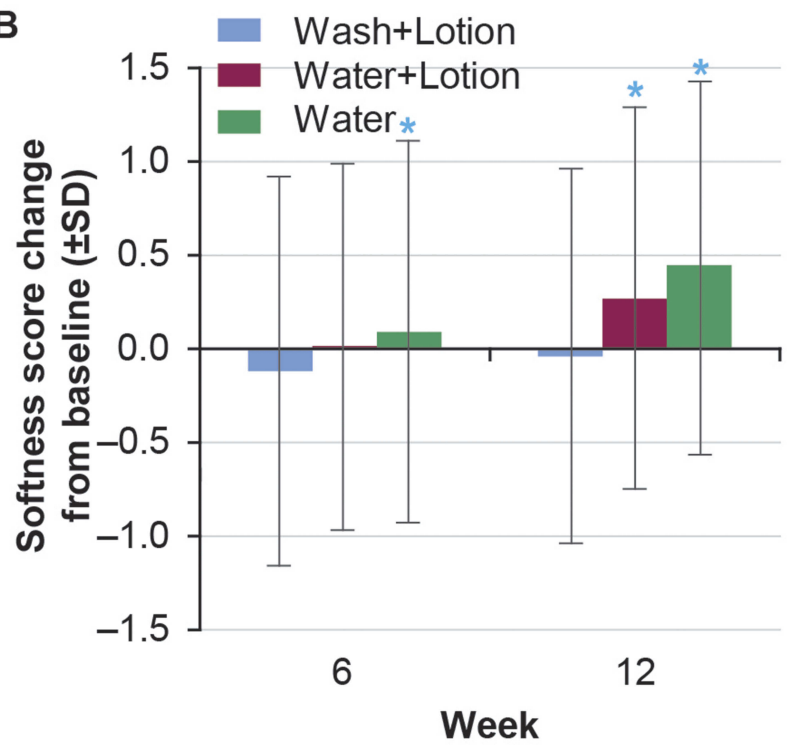

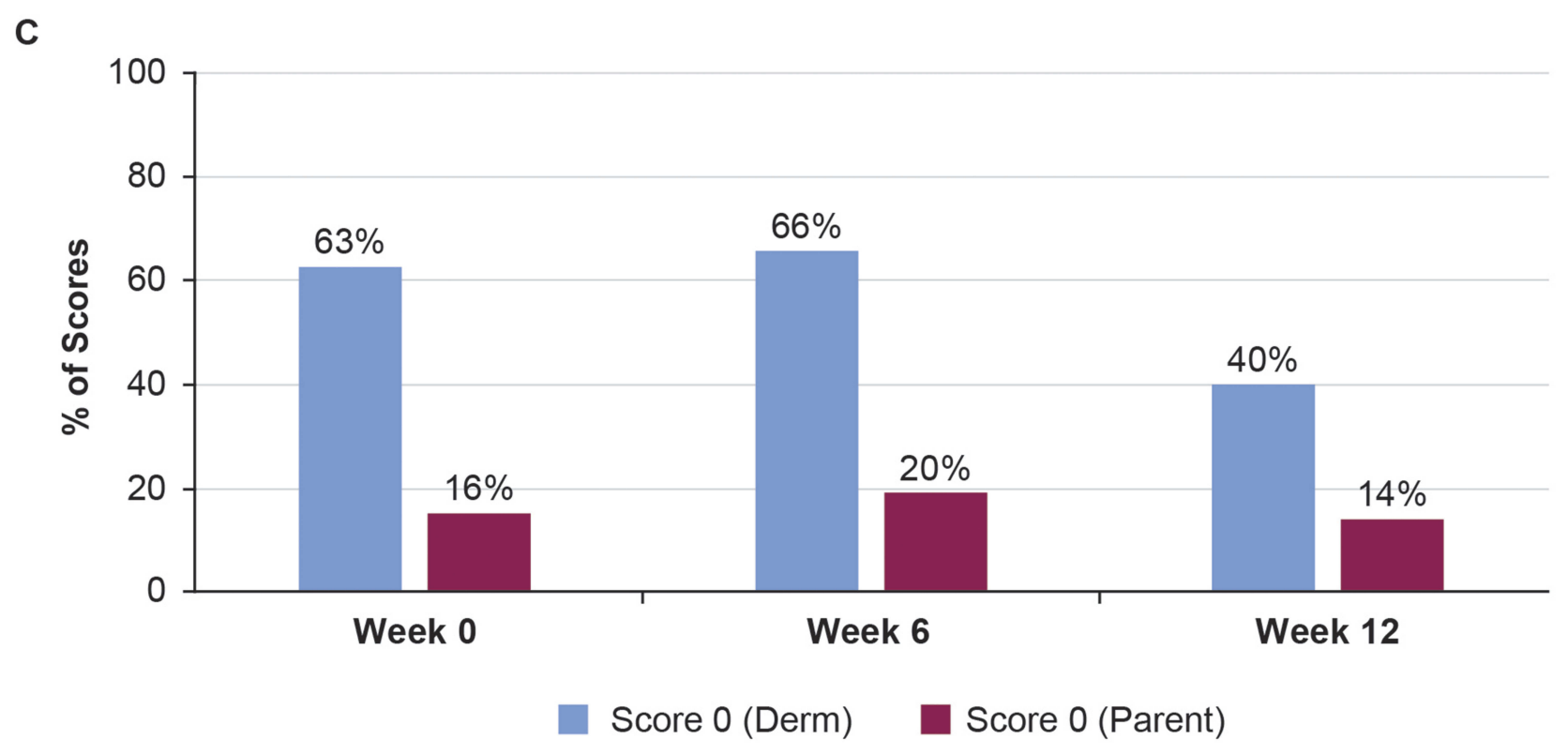

Figure 7 Skin softness. Dermatologist evaluation of skin softness score change from baseline (A). Parent/caregiver scores change from baseline (B). Comparison between dermatologist or parent scoring 0 at Week 0,6 and 12 (C).

Notes: $* P<0.05$. Color of asterisk over bar signifies the specific comparison made. Abbreviation: SD, standard deviation.

The cleanser and moisturizer used in this study were considered appropriate for use with babies based on being a $\mathrm{pH}$ neutral or mildly acidic cleanser that contains adequate and appropriate preservatives and surfactants, provides cleansing benefits without negatively altering the hydration property of skin, and does not cause irritation and alterations to skin surface $\mathrm{pH}$. For the moisturizer, appropriate was considered as formulated with mild and gentle ingredients and does not contain harmful substances that can be absorbed through baby skin.

This study has limitations that influence interpretation in addition to the seasonal changes affecting skin condition. Due to the difficulty of recruitment, the range of ages of the enrolled babies at the start of the study (aged 27-192 days, approximately 1-6 months) was wider than we initially planned (a more focused age range, aged 35-42 days). 


\section{Dermatologist}

A

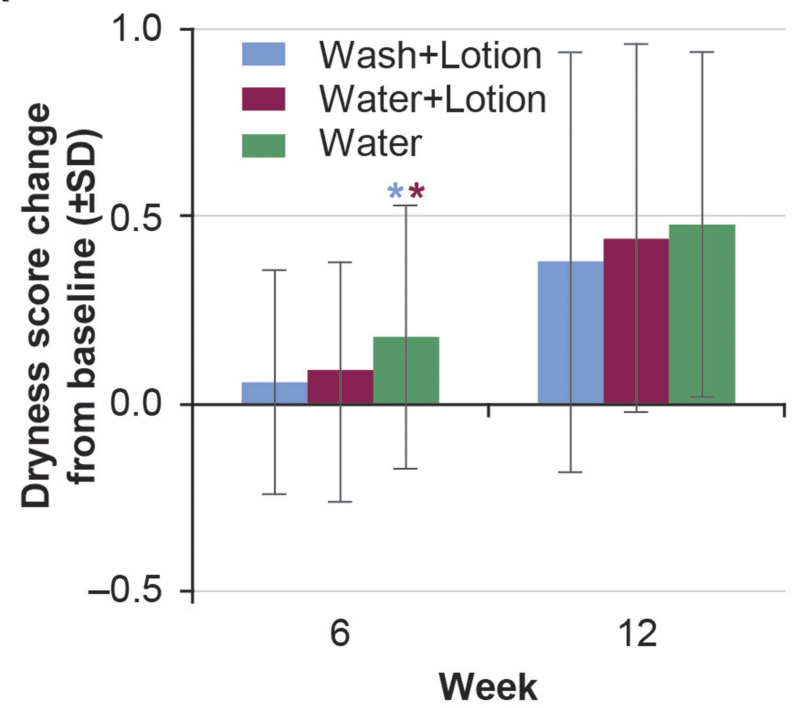

Parent/Caregiver

B

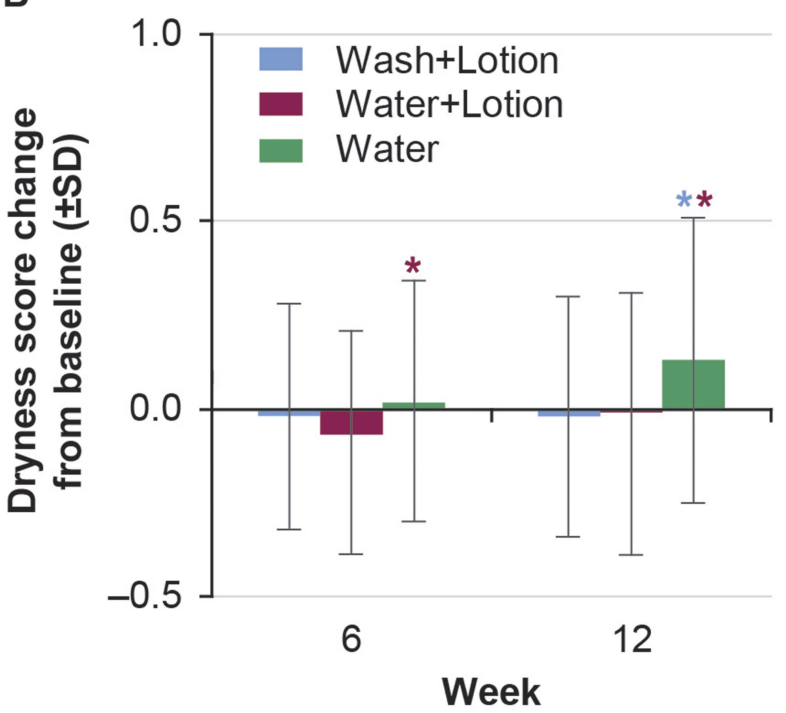

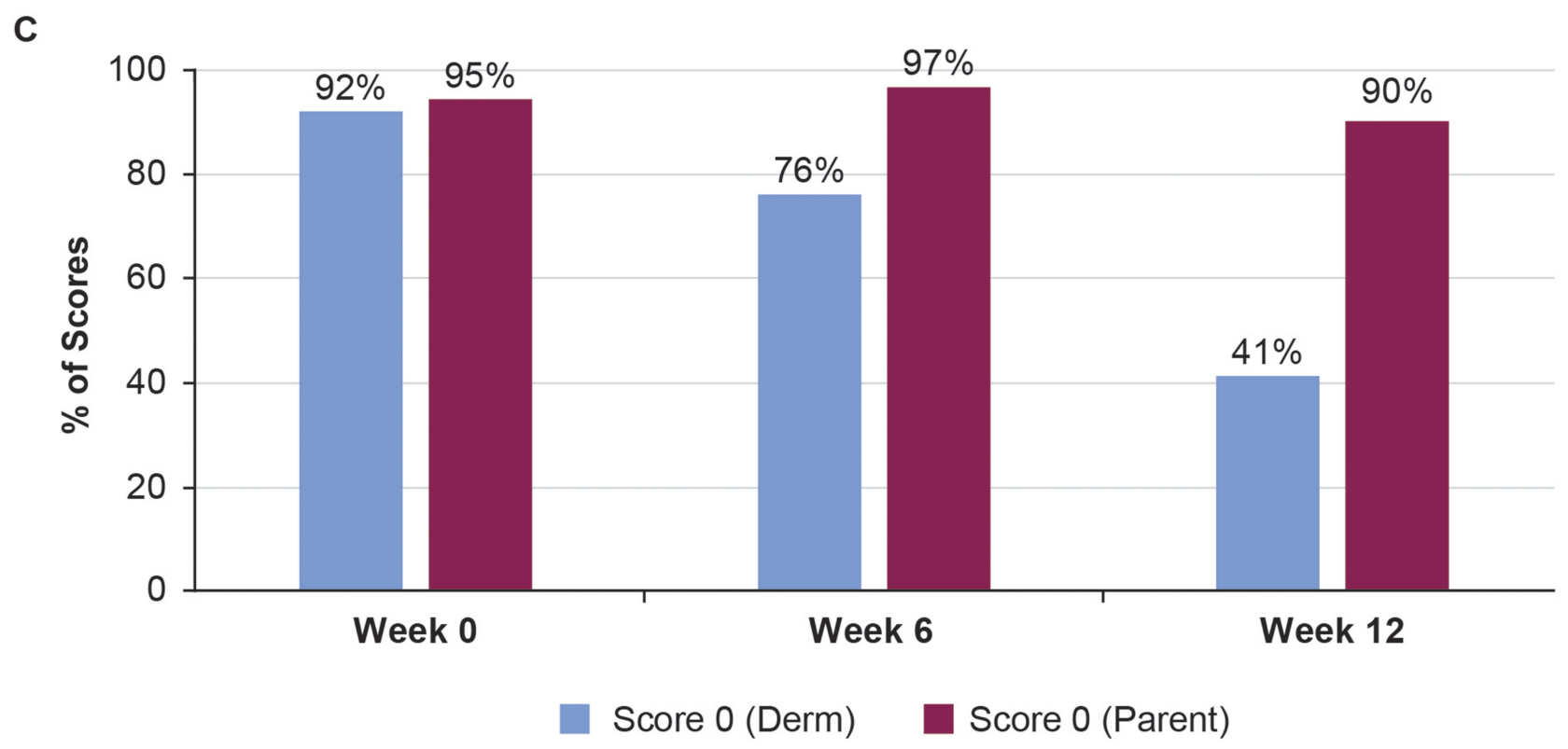

Figure 8 Skin dryness. Dermatologist evaluation of skin dryness score change from baseline (A). Parent/caregiver scores change from baseline (B). Comparison between dermatologist or parent scoring 0 at Week 0, 6 and 12 (C).

Note: ${ }^{*} P<0.05$. Color of asterisk over bar signifies the specific comparison made.

Abbreviation: SD, standard deviation.

Considering the skin development with age, this may have introduced more variability than we had originally hoped from the more focused age range. Study recruitment was also exclusively from one region in China and all enrolled babies were of Chinese Asian race. No differences between white and African American groups were observed for either TEWL or conductance in a recent study, ${ }^{1}$ but without further understanding, our results may be limited to the Chinese cohort studied.
Despite the potential interference from baby growth, normal skin maturation, and environmental variables (seasonal change), the regimens including the wash+lotion or water+lotion tended to show less change over time than water alone. This suggests that a regimen of bathing with a mild baby cleanser, followed by a moisturizer developed especially for baby skin was beneficial to baby skin; the developing skin of babies was more resistant and skin 
Dermatologist

A

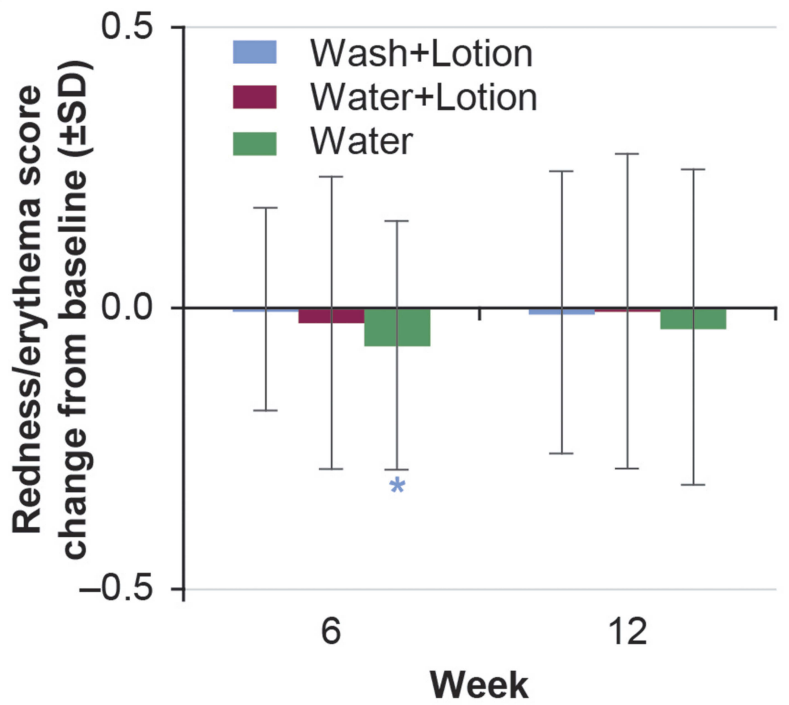

Parent/Caregiver

B

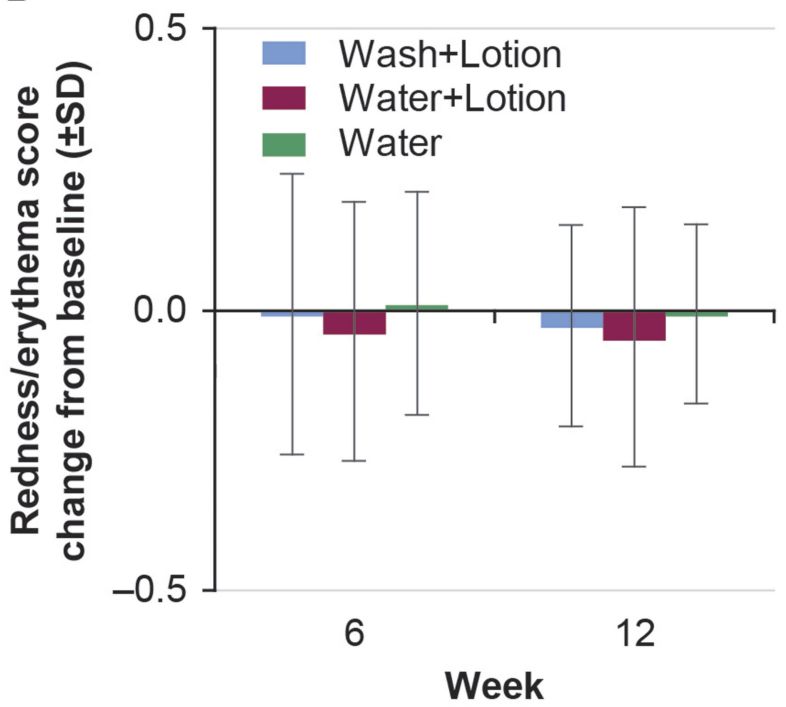

C

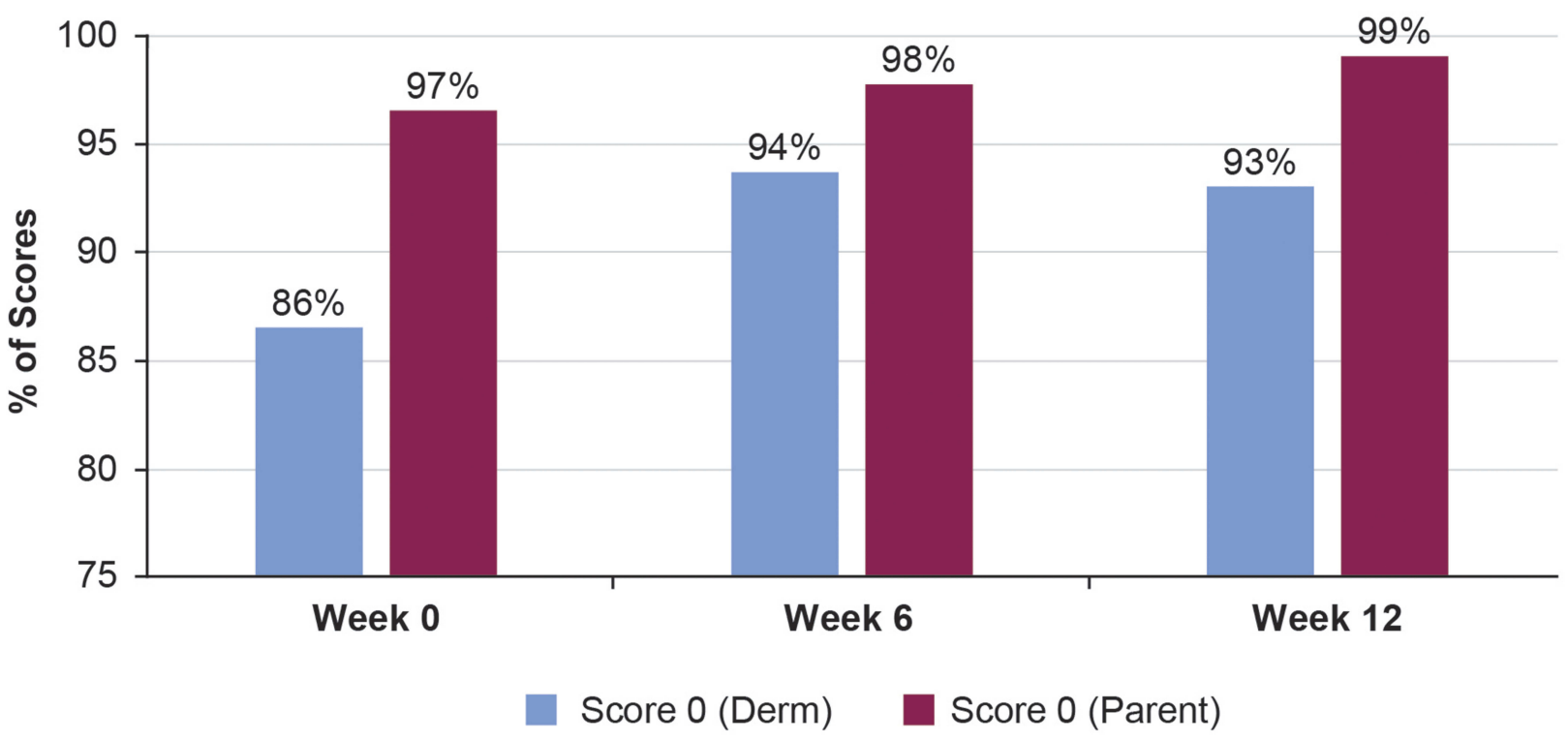

Figure 9 Redness/erythema. Dermatologist evaluation of redness/erythema score change from baseline (A). Parent/caregiver scores change from baseline (B). Comparison between dermatologist or parent scoring 0 at Week 0, 6 and 12 (C).

Note: $* P<0.05$. Color of asterisk over bar signifies the specific comparison made.

Abbreviation: SD, standard deviation.

conditions were maintained better or less worsened/ impacted by the seasonal challenges than without using any products (water only).

We believe this study reporting on skin parameters after using different skin care treatment regimens on very young babies provides valuable data to assist in understanding the potential benefits from washing with a specially formulated liquid baby cleanser and using baby lotion over an extended period. A recent systematic review of trials of shorter assessment duration to this study concluded that wash products had no evidence of significant differences compared with water and suggested a limitation of full-body emollients to high-risk babies with a genetic predisposition to eczema. ${ }^{14}$ In contrast, this 12 -week study in healthy babies with low-risk (from exclusion criteria) to eczema supports the inclusion of lotion in the skin care regimen. 


\section{Dermatologist}

A

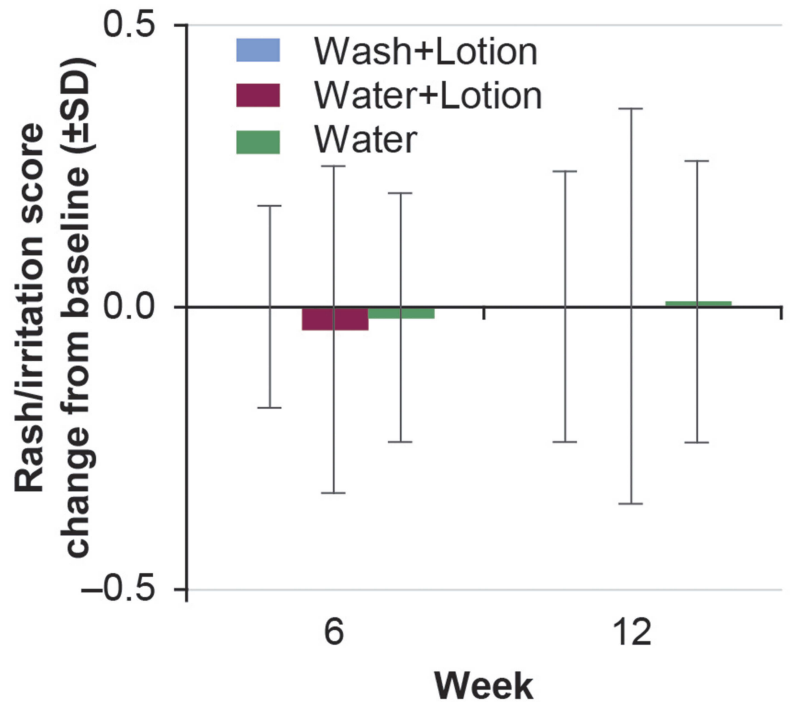

Parent/Caregiver

B

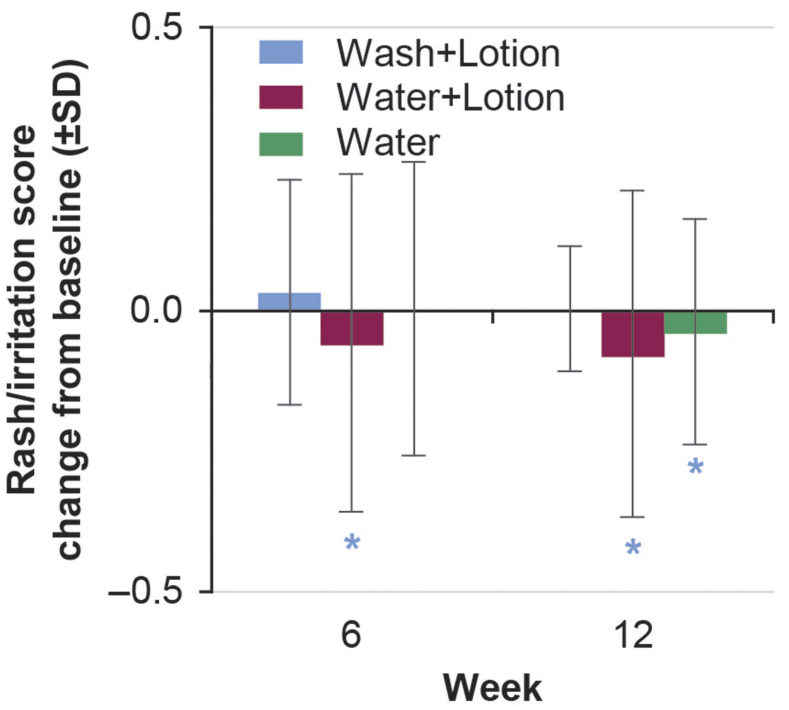

C

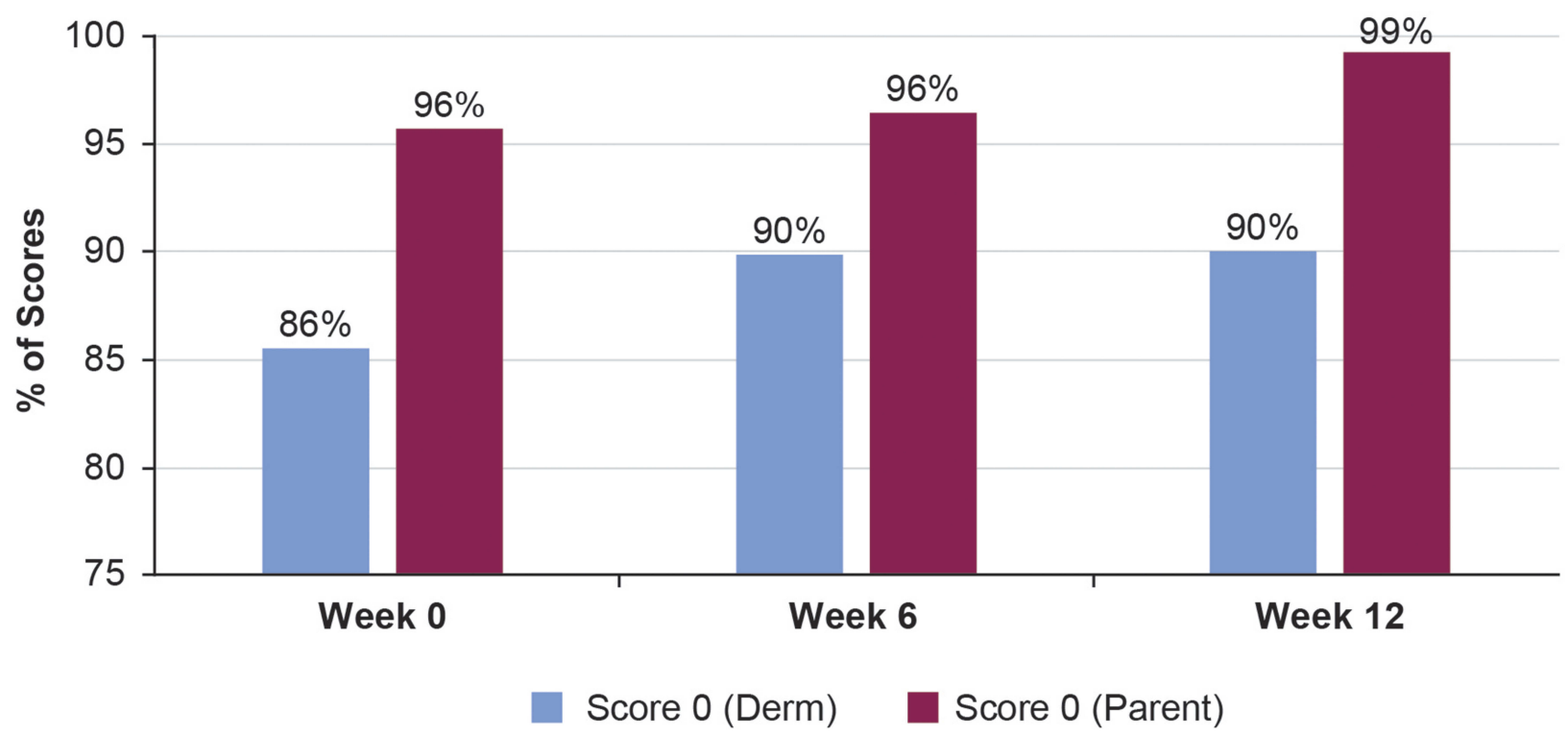

Figure 10 Rash/irritation. Dermatologist evaluation of rash/irritation score change from baseline (A). Parent/caregiver scores change from baseline (B). Comparison between dermatologist or parent scoring 0 at Week 0, 6 and 12 (C).

Note: $* P<0.05$. Color of asterisk over bar signifies the specific comparison made.

Abbreviation: SD, standard deviation.

Although these studies were conducted with healthy infants, more studies could be conducted to measure the tolerability and efficacy of the skin care regimens on infants with compromised skin (e.g., atopic dermatitis, eczema).

\section{Conclusion}

In summary, although skin condition deteriorated in all groups from seasonal effects, the instrumental findings and clinical evaluations demonstrated that bathing infants using a regimen of a liquid baby wash and a baby skin lotion resulted in better maintenance of skin moisture content, skin barrier function, skin $\mathrm{pH}$, and clinical characteristics than washing with water+lotion or water alone. Using an appropriately formulated, mild baby wash and baby skin lotion permitted effective cleansing without compromising skin health. 


\section{Dermatologist}

A

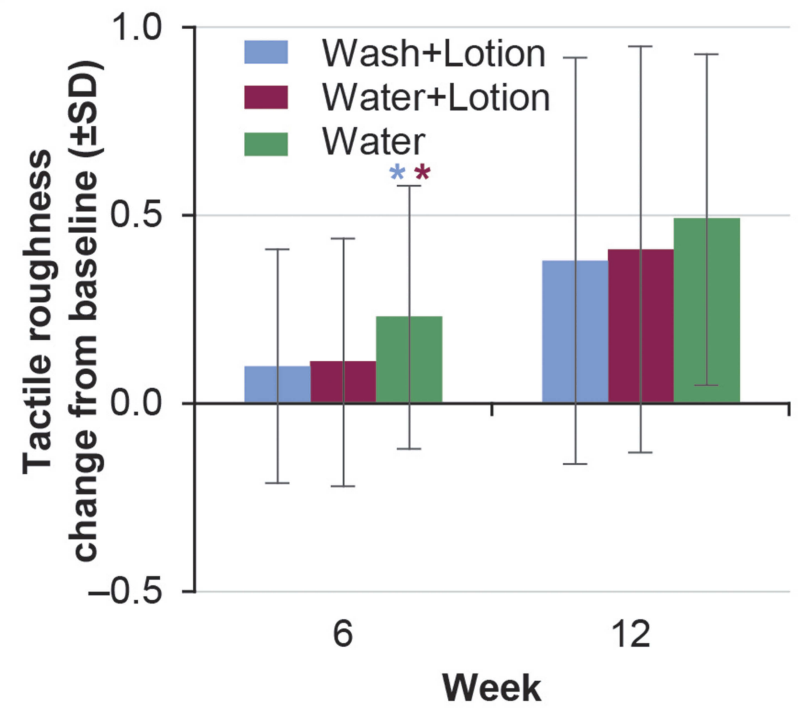

Parent/Caregiver

B

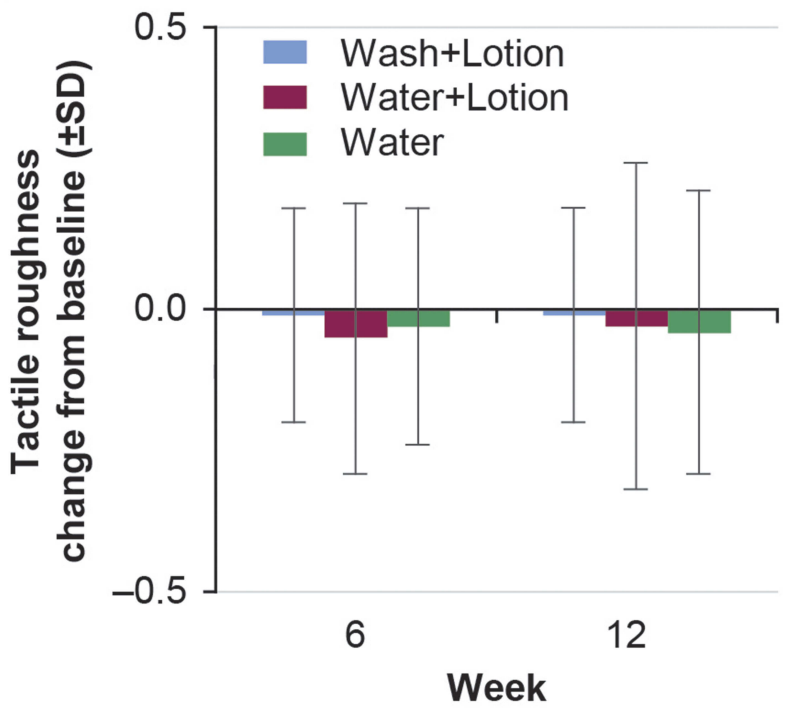

C

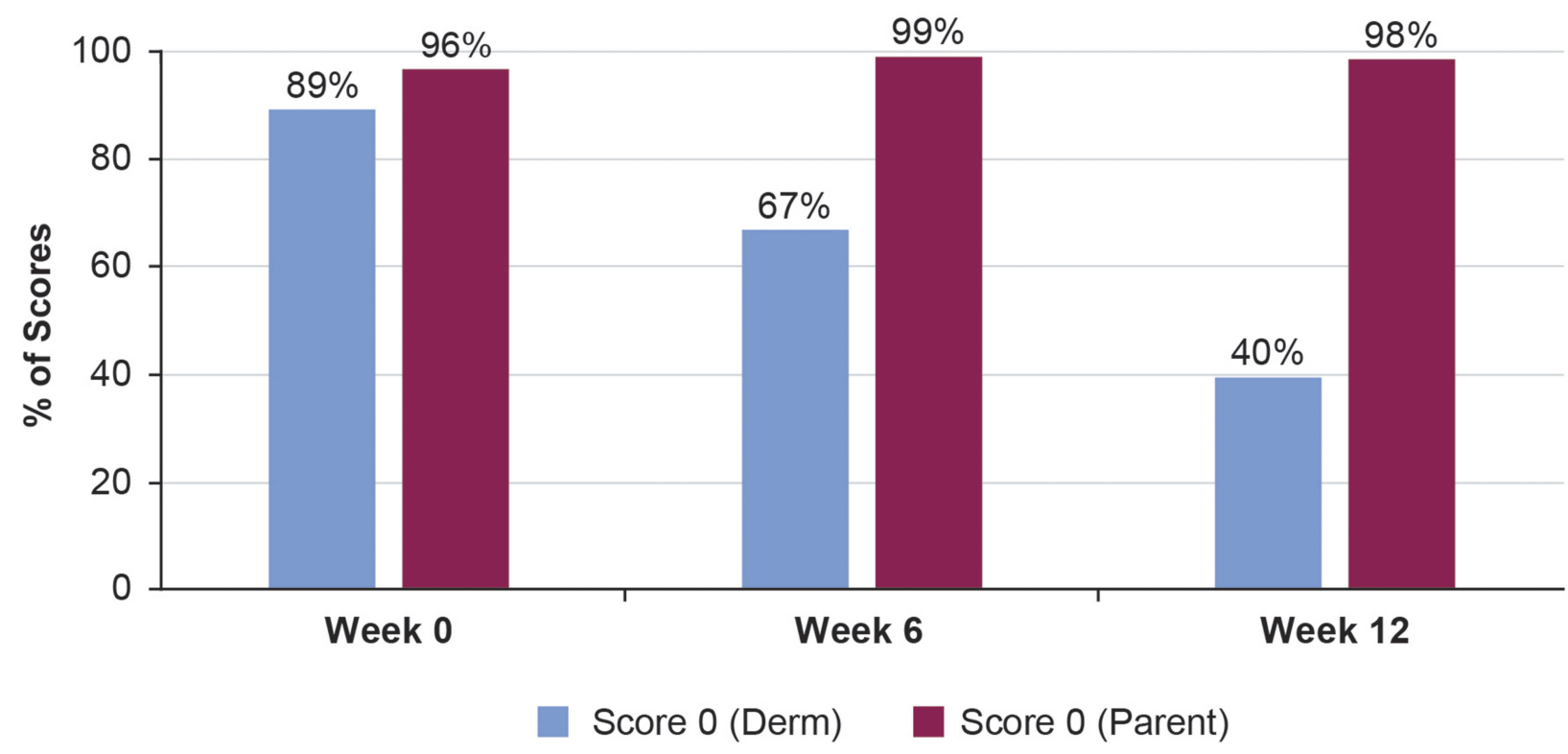

Figure I I Tactile roughness. Dermatologist evaluation of tactile roughness score change from baseline (A). Parent/caregiver scores change from baseline (B). Comparison between dermatologist or parent scoring 0 at Week 0, 6 and 12 (C).

Note: $* P<0.05$. Color of asterisk over bar signifies the specific comparison made.

Abbreviation: SD, standard deviation.

\section{Data Sharing Statement}

Any requests for data sharing should be submitted through the corresponding author, Carlos Galzote, at cgalzote@its.jnj.com.

\section{Acknowledgments}

The authors wish to thank the families of the study subjects for participating in this study. The authors want to thank Yujin Saito from Johnson \& Johnson International
Pte. Ltd., as he was part of the team conceiving the project. Medical writing and editorial support were provided by David Macari, PhD and Alex Loeb, PhD, CMPP, for Evidence Scientific Solutions, Inc. (Philadelphia, PA) and was funded by Johnson \& Johnson Consumer Inc. (Skillman, NJ). The authors also want to thank $\mathrm{Xi} \mathrm{Li}$ from Johnson \& Johnson AP Skin Testing Center and Georgios Stamatas from Johnson \& Johnson France for 
helpful comments as well as Min Qiu and Yanwen Jiang from Shanghai China-Norm Management Consulting CO., LTD, who provided statistical analysis support.

\section{Author Contributions}

All authors contributed to data analysis, drafting and revising the article, gave final approval of the version to be published, and agree to be accountable for all aspects of the work.

\section{Funding}

This study was funded by Johnson \& Johnson International Pte. Ltd (Singapore).

\section{Disclosure}

YYD, CG, and F-QK were employees of Johnson \& Johnson at the time this study was conducted. YYD and F-QK are no longer employed at Johnson \& Johnson. C-PS and LM received research support in association with this study. The authors report no other conflicts of interests in this work.

\section{References}

1. Mack MC, Chu MR, Tierney NK, et al. Water-holding and transport properties of skin stratum corneum of infants and toddlers are different from those of adults: studies in three geographical regions and four ethnic groups. Pediatr Dermatol. 2016;33(3):275-282.

2. Walters RM, Khanna P, Chu M, Mack MC. Developmental changes in skin barrier and structure during the first 5 years of life. Skin Pharmacol Physiol. 2016;29(3):111-118.

3. Nikolovski J, Stamatas GN, Kollias N, Wiegand BC. Barrier function and water-holding and transport properties of infant stratum corneum are different from adult and continue to develop through the first year of life. J Invest Dermatol. 2008;128(7):1728-1736.

4. Walters RM, Fevola MJ, LiBrizzi JJ, Martin K. Designing cleansers for the unique needs of baby skin. Cosmetics Toiletries. 2008;123(12):53-60.

5. Ananthapadmanabhan KP, Yu KK, Meyers CL, Aronson MP. Binding of surfactants to stratum corneum. J Soc Cosmet Chem. 1996;47:185-200.

6. Gfatter R, Hackl P, Braun F. Effects of soap and detergents on skin surface $\mathrm{pH}$, stratum corneum hydration and fat content in infants. Dermatology. 1997;195(3):258-262.

7. Blume-Peytavi U, Lavender T, Jenerowicz D, et al. Recommendations from a European roundtable meeting on best practice healthy infant skin care. Pediatr Dermatol. 2016;33(3):311-321.

8. Blume-Peytavi U, Hauser M, Stamatas GN, Pathirana D, Garcia Bartels N. Skin care practices for newborns and infants: review of the clinical evidence for best practices. Pediatr Dermatol. 2012;29 (1):1-14.
9. Garcia Bartels N, Scheufele R, Prosch F, et al. Effect of standardized skin care regimens on neonatal skin barrier function in different body areas. Pediatr Dermatol. 2010;27(1):1-8.

10. Dizon MV, Galzote C, Estanislao R, Mathew N, Sarkar R. Tolerance of baby cleansers in infants: a randomized controlled trial. Indian Pediatr. 2010;47(11):959-963.

11. Blume-Peytavi U, Cork MJ, Faergemann J, Szczapa J, Vanaclocha F, Gelmetti C. Bathing and cleansing in newborns from day 1 to first year of life: recommendations from a European round table meeting. J Eur Acad Dermatol Venereol. 2009;23(7):751-759.

12. Stamatas GN, Tierney N. Update on infant skin with special focus on dryness and the impact of moisturizers. In: Loden M, Maibach HI, editors. Treatment of Dry Skin Syndrome. Berlin: Springer-Verlag; 2012:295-310.

13. Horimukai K, Morita K, Narita M, et al. Application of moisturizer to neonates prevents development of atopic dermatitis. J Allergy Clin Immunol. 2014;134(4):824-830.e6. doi:10.1016/j.jaci.2014.07.060

14. Cooke A, Bedwell C, Campbell M, McGowan L, Ersser SJ, Lavender T. Skin care for healthy babies at term: a systematic review of the evidence. Midwifery. 2018;56:29-43.

15. Lund C. Bathing and beyond: current bathing controversies for newborn infants. Adv Neonatal Care. 2016;16(Suppl 5S):S13-S20.

16. Raboni R, Patrizi A, Cocchi G, Faldella G, Raone B. Comparison of two different neonatal skin care practices and their influence on transepidermal water loss in healthy newborns within first 10 days of life. Minerva Pediatr. 2014;66(5):369-374.

17. Lavender T, Bedwell C, O'Brien E, Cork MJ, Turner M, Hart A. Infant skin-cleansing product versus water: a pilot randomized, assessor-blinded controlled trial. BMC Pediatr. 2011;11:35.

18. Noviello MR; Italian Pediatric Group. Effects after daily use of washing products on infants aged $0-52$ weeks. Minerva Pediatr. 2005;57(6):411-418.

19. Atrux-Tallau N, Huynh NT, Gardette L, et al. Effects of physical and chemical treatments upon biophysical properties and micro-relief of human skin. Arch Dermatol Res. 2008;300(5):243-251.

20. Giusti F, Martella A, Bertoni L, Seidenari S. Skin barrier, hydration, and $\mathrm{pH}$ of the skin of infants under 2 years of age. Pediatr Dermatol. 2001;18(2):93-96.

21. Mayrovitz HN, Bernal M, Brlit F, Desfor R. Biophysical measures of skin tissue water: variations within and among anatomical sites and correlations between measures. Skin Res Technol. 2013;19(1):47-54.

22. Alanen E, Nuutinen J, Nicklén K, Lahtinen T, Mönkkönen J. Measurement of hydration in the stratum corneum with the MoistureMeter and comparison with the Corneometer. Skin Res Technol. 2004;10(1):32-37.

23. De Paepe K, Houben E, Adam R, Wiesemann F, Rogiers V. Validation of the VapoMeter, a closed unventilated chamber system to assess transepidermal water loss vs. the open chamber Tewameter ${ }^{\mathbb{R}}$. Skin Res Technol. 2005;11(1):61-69.

24. Fluhr JW, Darlenski R, Lachmann N, et al. Infant epidermal skin physiology: adaptation after birth. Br J Dermatol. 2012;166(3):483490.

25. Association of Women's Health Obstetric and Neonatal Nurses. Neonatal Skin Care: Evidence-Based Clinical Practice Guideline. 3rd ed. Washington, DC: Association of Women's Health, Obstetric and Neonatal Nurses; 2013.
Clinical, Cosmetic and Investigational Dermatology

\section{Publish your work in this journal}

Clinical, Cosmetic and Investigational Dermatology is an international, peer-reviewed, open access, online journal that focuses on the latest clinical and experimental research in all aspects of skin disease and cosmetic interventions. This journal is indexed on CAS.
The manuscript management system is completely online and includes a very quick and fair peer-review system, which is all easy to use. Visit http://www.dovepress.com/testimonials.php to read real quotes from published authors. 